\title{
Towards Robust Communications of Wireless Sensor Networks in Vehicular Environments: A Case Study
}

\author{
Silvia Krug, Hannes Toepfer, Tino Hutschenreuther and Jochen Seitz
}

\begin{abstract}
Wireless Sensor Networks are popular solutions for various applications in order to reduce the efforts required by traditional cable-based systems. If the system in question is used to monitor and control physical processes, robust and reliable communication services have to be provided by the network. This is especially true for industrial or vehicular applications where the nodes are deployed in environments facing harsh propagation conditions. Usually, the effect of this on the system performance is studied on isolated layers of the OSI-reference model only Therefore, we present results from a measurement campaign of vehicular environments that try to estimate the achievable robustness with respect to multiple OSI layers. Our results show that even if the link is rather poor, protocols on the upper layers are able to compensate this at the cost of additional delay.
\end{abstract}

Index Terms-Wireless Sensor Networks; Performance Evaluation; Testbed; Vehicular Environment.

\section{INTRODUCTION}

Vehicles and intelligent transportation systems (ITS) as well as other industrial applications can benefit from the deployment of wireless sensor networks (WSNs) to both monitor and control different processes. In case of ITS, such processes could be vehicular traffic flows or the operation of the vehicles themselves, if autonomous driving is the goal. However, the control loop requires robust and reliable data transmissions within certain time constraints and has to ensure that decisions are based on up-to-date data values [1]. This is true for both communication directions e.g. the sensor node to the control unit and then the control unit to the actuator where sensors and actuators could be attached to the same network node or different ones.

On the other hand, ITS or industrial environments pose some unique challenges on the signal propagation due to heavily metalized and obstructed surroundings. This is a major challenge for the required robust and error-free communication if wireless access technologies are used [2]. Several authors have already studied the impact of such environments on

Manuscript received May 24, 2017; revised September 29, 2017. Date of publication: December 13, 2017.

Silvia Krug and Jochen Seitz are with the Technische Universität Ilmenau, Germany. Hannes Toepfer is with the Technische Universität Ilmenau, Germany and with the IMMS Institute for Microelectronic and Mechatronic Systems not-for-profit GmbH, Ilmenau, Germany. Tino Hutschenreuther is with the IMMS Institute for Microelectronic and Mechatronic Systems notfor-profit $\mathrm{GmbH}$, Ilmenau, Germany.

E-mails:\{silvia.krug,jochen.seitz,hannes.toepfer\}@tu-ilmenau.de, tino.hutschenreuther@imms.de

Digital Object Identifier (DOI): 10.24138/jcomss.v13i4.364 different levels e.g. the pure radio propagation or the impact on medium access schemes. However, a system based on wireless sensor nodes will always incorporate a complete protocol stack and a desired user application defining the requirements on the overall end-to-end communication service provided by the complete network. In such a setup, several protocols are applied and each one is capable of handling failures to some extend. The overall quality of the communication is therefore not only depending on one isolated aspect. However, this multi-layer design to achieve reliable data transmission also results in additional delay which can be undesirable especially in control applications. Besides that, retransmissions or other mechanisms on protocol side require additional energy resulting in shorter operation time for battery-powered nodes. Due to these considerations, the employment of current wireless sensor nodes in critical components is limited by several trade-offs between communication requirements under difficult conditions as well as the energy budget and desired node lifetime. In conclusion there are already several studies on wireless sensor networks deployed within vehicles. However, all of them focus on individual aspects of the communication stack and do not try to assess the performance of the system and nodes from a cross-layer perspective. Therefore, we study the robustness of WSN communication deployed in vehicular environments based on various measurements and estimate the robustness of the communication service provided by the network to the deployed applications. We present insights from a measurement campaign characterizing the performance of traditional wireless sensor nodes operating on the IEEE 802.15.4 standard deployed in vehicular environments. The study is performed in-situ with custom multi-purpose sensor nodes. In contrast to other studies, we observe the robustness indicators on multiple layers by combining measurements of the received signal strength, the achievable success rates on the MAC and network layers as well as the experienced round trip delay at the network layer. This allows us to study the impact of faulty links on higher layer performance as well as the overall network reliability.

The goal is to evaluate the effects of the given channel characteristics on the sensor node system and how the configured network protocols perform under such conditions. Instead of studying the channel characteristics only, we want to understand what type of service the network is able to provide to any application under difficult environmental conditions. 
Based on our results, we analyze the trade-off between robustness provided by various protocols and the timing constraints of control applications and discuss further enhancements to leverage this effect. Even if a simplistic scenario is chosen, it already reveals some problems in such an environment that require further research in order to build robust and reliable intra-vehicle sensor networks.

The paper is organized as follows. First, we review existing work and discuss similar measurement studies in section II. In Section III, we introduce the customized sensor node platform used for the evaluation. Afterwards, we present the setup and the results of our measurement campaign and discuss them in detail in Section IV. Finally, the paper is concluded in Section $\mathrm{V}$ including a number of possible future research questions.

\section{RELATED WORK}

Several authors discuss options to employ wireless sensor nodes either within vehicles [3] or other industrial application domains [4], [5] featuring similar harsh propagation characteristics due to metalized surroundings. These papers in general discuss remaining challenges and evaluate potential issues using simulations or analytical approaches only. Ideally, this requires a first set of measurements in order to characterize the target environment and then to derive the necessary constraints. Besides these papers discussing the general applicability of sensor networks in industrial or vehicular environments, some studies have focused on experimental evaluation of these networks.

The focus of the work presented in [6] is to identify coexistence issues between different network access technologies. To do that the authors use a testbed, that allows them to merge signals from different sources and attenuate them accordingly by collecting the signal from the antenna connector of the transceivers in question. While this gives good insights on theoretical overlaps, it does not reflect effects that come from multipath reflections and further noise sources.

Another approach that is more realistic is presented in [7]. There, the authors characterize an indoor scenario using ZigBee devices. The results are however not applicable for heavily metalized environments such as vehicles. In [8], the authors studied the impact of electromagnetic interference using a similar radio chip in real world industrial deployments. Their results show that wireless sensor networks can work well in metalized environments, but the type of interference can have a significant impact. The study is meant as an indication of how to design and install similar networks. However, the results were derived for industrial settings with rather large distances between sender and receiver. While the cluttered metalized environment is similar, the distances will be smaller in vehicular settings and thus the results cannot be applied one on one to the other environment.

The study presented in [9] was performed within vehicles showing the effect of interference from other network access technologies on ZigBee nodes. While the impact of the interference is clearly described, the base performance of each network technology remains open as well as connectivity issues caused within one technology. Besides that, the authors use only one sender and receiver pair per technology at selected locations and thus their results might be position dependent.

Other works focus on the topology of sensor node deployments within vehicles as for example presented in [10]. They measure again interference, between sensors and $\mathrm{WiFi}$ nodes within a vehicle and show that multi-hop topologies can achieve a better performance. But this study does not focus on the characterization of the communication either.

\section{SENSOR PlatForm}

For our experiments, we use a custom sensor node platform which was developed at the IMMS as part of the BASeNet [11] architecture. Therefore, we will first introduce both the hardware and the software components of the nodes in question.

\section{A. Hardware}

The development of our hardware was inspired by Iris Motes and the particular design goal was to develop a multipurpose sensing platform. Table I gives the main characteristics of the nodes.

TABLE I

HARDWARE CHARACTERISTICS

\begin{tabular}{ll}
\hline Parameter & Value \\
\hline Controller & ATMega1281 \\
Clock Speed & $8 \mathrm{MHz}$ \\
RAM & $10 \mathrm{kByte}$ \\
ROM & $48 \mathrm{kByte}$ \\
\hline Transceiver & RF230 \\
\hline Power Supply & 2 AA Batteries / USB \\
\hline
\end{tabular}

The employed Radio Frequency (RF) transceiver supports default 802.15.4 Physical (PHY) and Medium Access Control (MAC) layers and operates at $2.4 \mathrm{GHz}$. This transceiver has a sensitivity threshold of $-91 \mathrm{dBm}$ for the Received Signal Strength Indicator (RSSI) and provides RSSI measurements in steps of $3 \mathrm{dBm}$.

Figure 1 shows an example node with and without casing. The Serial-to-USB communication interface to connect one node to a personal computer (PC) is also visible in Figure $1 \mathrm{~b}$.

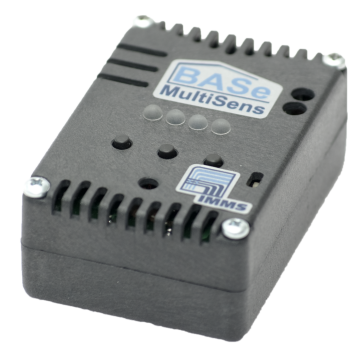

(a) with casing

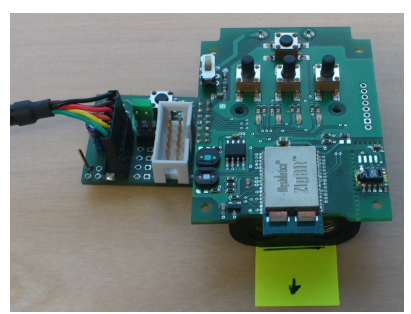

(b) bare board
Fig. 1. Used hardware for experiments 
To build a multi-purpose platform, we equipped each node with a multitude of sensors, e.g. to measure luminance, temperature, humidity, and $\mathrm{CO}_{2}$. Besides that, the nodes support switchable mains plugs, general-purpose reed contacts, and freely configurable general purpose input output pins as a possibility to integrate customer-specific sensors.

\section{B. Software}

The nodes are operated with a customized version of TinyOS [12] that was adapted to support our hardware platform and its default sensor configuration.

Usually, components of the investigated BASe-Net system communicate via IPv6, using proprietary application-level protocols [13], which are encapsulated in 6LoWPAN. Nodes form a tree-like network topology with a central gateway node. Possible intermediate nodes are sensor nodes with additional routing capabilities.

Multi-hop routing is allowed based on the BLIP implementation of the HYDRO algorithm [14] in TinyOS. However, multi-hop capabilities are not necessarily required for the measurements presented here because the nodes are placed in close proximity to each other (cf. Section IV for the detailed setup). The nodes are mostly forming a star-topology in this case. Multi-hop capabilities were still enabled in order to provide additional robustness by offering multiple communication paths.

To evaluate the performance available for applications we employed the UDPEcho application on all sensor nodes and the IPBaseStation application on the gateway node. This setup includes the complete communication stack up to the transport layer. UDPEcho provides ICMP features to each node, allowing the node to answer and initiate ICMP-EchoRequests similar to ping6 on Linux machines, but with a limited set of configuration options. This allows the usage of default ICMPtools such as ping6 for the evaluation of delay and delivery ratio. Ping6 sends a predefined number of packets at a given sending interval to a destination node and records the time between sending the request and receiving the answer as round trip delay for each packet. Besides that, it reports the number of duplicated and lost packets.

To evaluate the link and propagation characteristics within the car, we use a different approach. The employed radio transceiver is able to capture the RSSI value for every packet. Therefore, we use a custom TinyOS application to generate small messages at a configurable transmission rate on each sensor node. At the gateway node, we report the received RSSI values. This application directly accesses the link layer as provided by the platform's transceiver and does not apply any error correction or message repetition mechanisms. Experiments with these test applications allow the evaluation of given link characteristics and their impact on higher layers of the communication stack.

\section{Measurement Campaign}

This section describes the measurement setup used for the evaluation, provides details on the various experiments and presents our results.

\section{A. Measurement Setup}

We performed measurements in a Peugeot 208 by placing up to 10 sensor nodes in the interior or exterior of the car forming two separate sensor subsystems. One subsystem (square, green) is placed in the cars interior with positions on all seats that might be used to sense whether seats are occupied by passengers or used for air conditioning / seat heating. The second exterior subsystem (circles, red) uses sensor nodes that are placed inside each wheel. These nodes are not tire sensors but are placed at the inside (pointing towards the axle) of the wheel rim. Figure 2 shows the positions in top view. The gateway (diamond, blue) is placed at the center of the dashboard.



Fig. 2. Schematic illustration of the node placement within the car

The antennas of the nodes belonging to the internal subsystem are directed towards the gateway node, facing forward in driving direction of the car. In case of the external subsystem, all antennas point to the opposite wheel. The gateway node $(\mathrm{GW})$ is connected via USB to a measurement personal computer (PC), as shown in Figure 3. These two devices act as one component, the base station, and thus the serial connection as well as the required drivers add additional delay to any delay measurement initiated or measured at the PC. Using a combination of the UDPEcho application and the default ping6 tool on the PC allows to measure three different delay values, that are also presented in Figure 3.

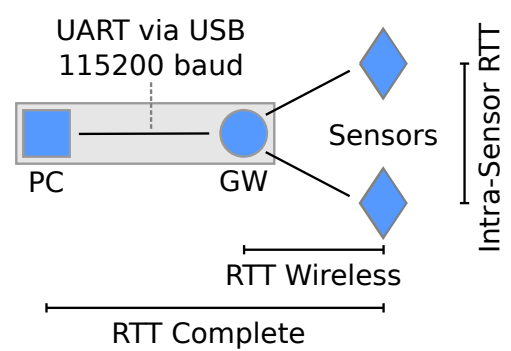

Fig. 3. Measurement concept with a base station and multiple sensor nodes

The complete Round Trip Time (RTT) applies to all measurements initiated by the PC. It consists of the wireless part between sensor nodes and the gateway as well as the delay introduced by the connection to the control PC. UDPEcho allows the initiation of ICMP-Requests and thus allows an innetwork measurement of the delay between the sensor nodes themselves. Due to the limited configuration options of the 
application, this is however limited to the default settings of ping6, i. e. a sending interval of 1 second, 56 Byte payload, and 10 packets per execution of the command. Unfortunately, this allows a basic evaluation of the delay under low traffic loads, only. Higher traffic loads could potentially result in further congestion or packet collisions. This was tested by placing multiple requests at the same time in one use case. Further tool enhancements are required to generate arbitrary traffic loads for these evaluations.

\section{B. Experiments}

Based on the setup introduced in the previous section, we performed several measurements to evaluate the performance of our nodes in vehicular environments. All measurements were performed on a parking lot without any other vehicles within a range of about $50 \mathrm{~m}$, a driver on its seat, and with closed doors. The engine as well as other electronics were switched off during the measurements. These conditions create an idealized scenario which is rather simple as additional more realistic features such as working engines or other cars are missing. However, it is suitable to study the performance of the network and the propagation effects within the car to identify what is possible in good conditions. More realistic scenarios are expected to show a worse performance due to more complex propagation conditions.

In order to evaluate the channel characteristics, we first performed measurements to capture the RSSI value of received packets and evaluated the potential packet loss. In this setup, the nodes start sending small packets as soon as they are switched on and the base station collects this information once the IP driver is configured and setup. Afterwards, we performed a second set of measurements using ping6 in order to evaluate the impact of the link characteristics on higher layer protocols and thus applications. Table II gives an overview of the different setups.

TABLE II

MEASUREMENT SETUP

\begin{tabular}{ll}
\hline \multicolumn{1}{c}{ Parameter } & Value \\
\hline General & \\
\hline Number of nodes & 10 \\
Channel & 12 \\
Tx Power & $3 \mathrm{dBm}$ \\
\hline RSSI & \\
\hline Sending interval & $0.1 \mathrm{~s}$ \\
Duration & $2 \mathrm{~min}$ \\
\hline Ping6 Measurements & \\
\hline Sending intervals & $1,0.5,0.2$, and $0.1 \mathrm{~s}$ \\
Number of packets & 500,1000, and 1500 packets \\
Data payload & $56 \mathrm{Byte}$ \\
\hline
\end{tabular}

During the measurements with ping6, we changed and, in case of the sensor nodes, permuted the node initiating the requests as described in Table III in order to evaluate all three options described in Figure 3.
TABLE III

PING6 SETUPS

\begin{tabular}{ll}
\hline Initiator & Target \\
\hline PC & all sensors (single) \\
& all sensors in parallel \\
\hline Sensor & all sensors and GW (single) \\
& all sensors and GW routers in parallel \\
\hline
\end{tabular}

\section{Results and Discussion}

First, we analyzed the RSSI values retrieved for each position. Figure IV-C presents the corresponding results for measurements of the two subsystems individually and all nodes combined.

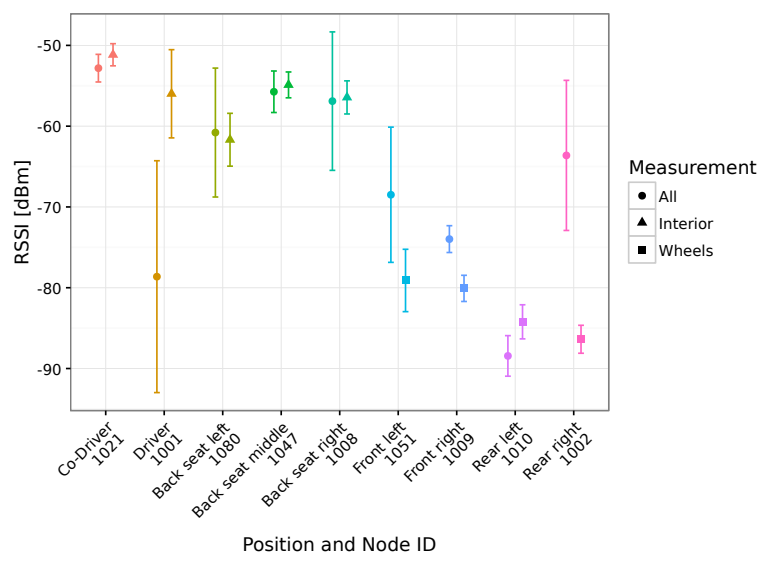

Fig. 4. Average RSSI values and variance of the nodes shown in Figure 2 from two subsystems measured at the PC component of the base station for two measurement setups per node

As expected, the interior subsystem shows good values around $-55 \mathrm{dBm}$ while the wheel subsystem shows a reduced signal strength of about $-82 \mathrm{dBm}$ due to the node positions at the exterior of the car and thus potential blockage by the body of the car. Surprisingly, even the outdoor nodes show almost no packet loss if evaluated separately even though their RSSI value is close to the receiver threshold $-91 \mathrm{dBm}$ and thus indicating rather poor propagation conditions.

However, the values show large variations, if both systems are used in parallel. The significant drop for the node placed at the driver seat and the increased value for rear right wheel are rather unexpected and suggest some form of interference.

While these first measurements show promising results, one should keep in mind that the conditions were rather ideal. For moving vehicles, additional electronic devices, or vehicles with passengers, we expect worse performance. This can be crucial especially for the exterior subsystem, since the RSSI values of those nodes are already quite close to the receiver sensitivity limits.

While RSSI is a good indicator for the general reception probability of nodes, it does not reflect whether connections are possible at higher layers. To get information about this, we studied the delivery ratio and round trip time (RTT) based on ping6 evaluations. We performed measurements for each subset separately as well as for all nodes combined. First, 


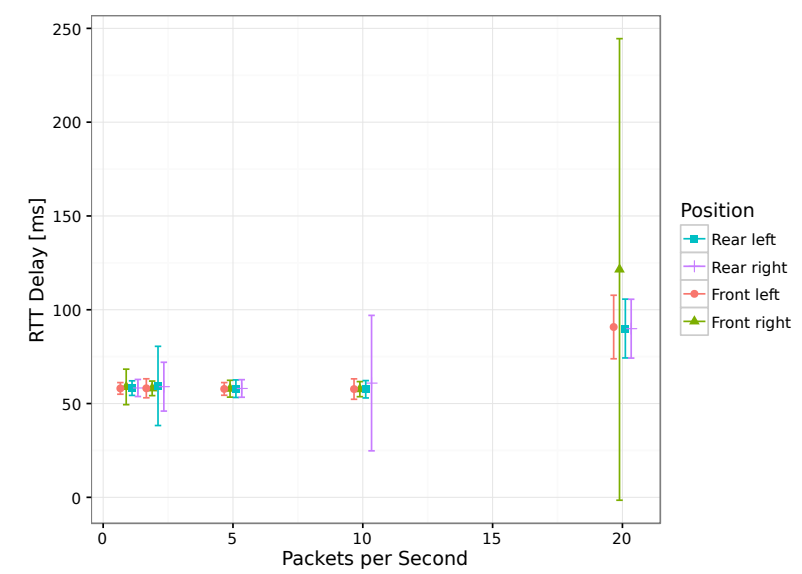

(a) Wheel subsystem

Fig. 5. Average RTT values

we used the PC to introduce the traffic to the network using various sending intervals as supported by ping6 on Linux operating systems. The results thus represent the RTT complete values as introduced in Figure 3. Figure 5 presents the corresponding results for measurements of the two subsystems individually. Each individual measurement point represents a set of individual samples with quite rough spacing that do not allow for any interpolation between points.

Again, the packet loss for all measurements is small $(<1 \%)$ and thus not presented separately. When comparing the different packet generation rates, we notice that the delay remains the same for the first four measurement points, while it is significantly higher for the fifth one. The values reported for the first four sets are somewhat higher than expected, potentially caused by the additional delay required for the transfer of the packet from the PC via the serial connection to the actual transmitting gateway node. This is also the reason for the higher RTT value for the fifth measurement configuration with a data rate of 20 packets per second, because the serial connection turns out to be a bottleneck for the communication in this case. Future work will include measurements with finer sampling within the range of the fourth and fifth measurement point to study this in more detail.

When trying to reach all nodes of one subsystem in parallel by placing multiple calls to ping6 in separate terminal instances, the observed effect becomes more severe, as presented in Figure IV-C for the wheel subsystem. The result is merely a higher traffic load and not a truly parallel communication, but the nodes are competing for available channel resources in this case.

In Figure IV-C, we plotted the RTT as presented above using a logarithmic axis for the delay values. Communicating with all four nodes in parallel is increasing the load on the network. While this is not a significant problem for data rates up to 20 packets per second, the delay is increased to an average of around $12 \mathrm{~s}$ for higher data rates. Still the packet loss is low, but the delay is unacceptable for most applications. This analysis on system level clearly indicates that a gateway connecting the sensor nodes to the traditional intra-vehicle

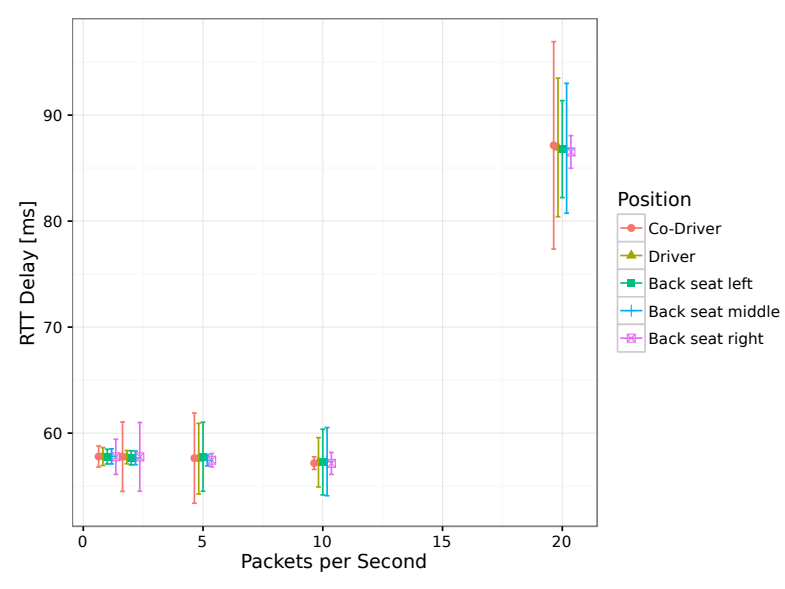

(b) Interior subsystem

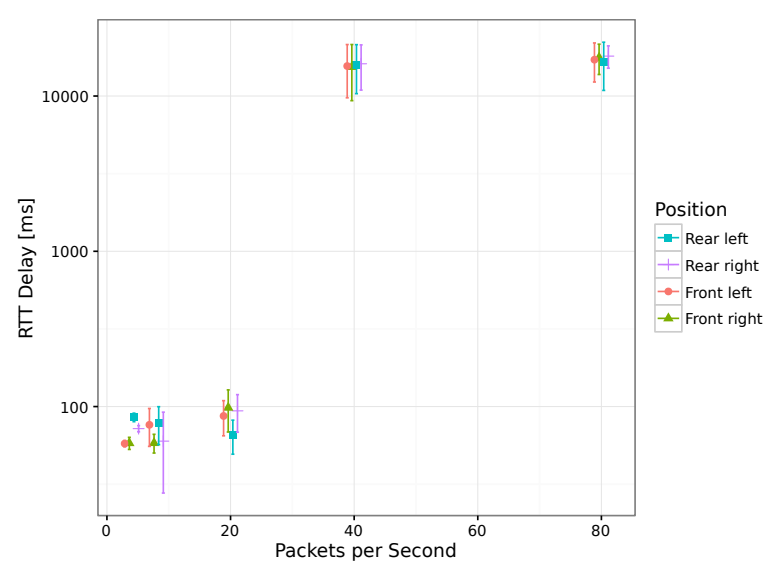

Fig. 6. Average RTT values from wheel subsystem - polling all nodes in parallel

network has to be designed with the appropriate throughput in mind.

In order to better estimate the delay for the wireless transmissions only, we performed another set of measurements based on the UPDEcho application that comes with TinyOS and supports ping-like functionality from within the network, without the need to pass messages through the serial connection to the PC. Unfortunately, this application does not provide the same parameters as ping6 so that we were only able to mimick the default settings with 1 packet per second and a total sending interval of $10 \mathrm{~s}$. But compared to the previous measurement with ping6 on the $\mathrm{PC}$, this application allows to ping all link-local nodes via IPv6 by using the corresponding multicast address ( $f f 02:: 1$ ). This results in broadcast requests, answered by all nodes in the surrounding. Consequently, when permuting the sending node, we are able to obtain values for all links in both directions, where a given node is once the sender of the requests and destination of the requests in all other cases. Table IV shows the corresponding average RTT values and Table V shows the corresponding error rates between any two nodes. 
TABLE IV

In-Network Delay fOR ALl Node Permutations

\begin{tabular}{crrrrrrrrr}
\hline dest & \multicolumn{70}{c}{ source } \\
\cline { 2 - 10 } node ID & 1001 & 1002 & 1008 & 1009 & 1010 & 1021 & 1047 & 1051 & 1080 \\
\hline 1001 & - & $150.89 \mathrm{~ms}$ & $85.80 \mathrm{~ms}$ & $83.50 \mathrm{~ms}$ & $139.50 \mathrm{~ms}$ & $78.83 \mathrm{~ms}$ & $72.00 \mathrm{~ms}$ & $239.00 \mathrm{~ms}$ & $83.50 \mathrm{~ms}$ \\
1002 & $186.63 \mathrm{~ms}$ & - & $133.00 \mathrm{~ms}$ & $98.60 \mathrm{~ms}$ & $47.37 \mathrm{~ms}$ & $133.27 \mathrm{~ms}$ & $116.30 \mathrm{~ms}$ & $239.30 \mathrm{~ms}$ & $118.00 \mathrm{~ms}$ \\
1008 & $112.92 \mathrm{~ms}$ & $136.44 \mathrm{~ms}$ & - & $114.20 \mathrm{~ms}$ & $83.75 \mathrm{~ms}$ & $70.00 \mathrm{~ms}$ & $57.30 \mathrm{~ms}$ & $227.88 \mathrm{~ms}$ & $88.13 \mathrm{~ms}$ \\
1009 & $70.50 \mathrm{~ms}$ & $91.44 \mathrm{~ms}$ & $78.67 \mathrm{~ms}$ & - & $97.50 \mathrm{~ms}$ & $86.00 \mathrm{~ms}$ & $129.70 \mathrm{~ms}$ & $210.11 \mathrm{~ms}$ & $118.00 \mathrm{~ms}$ \\
1010 & $159.08 \mathrm{~ms}$ & $74.33 \mathrm{~ms}$ & $108.90 \mathrm{~ms}$ & $40.73 \mathrm{~ms}$ & - & $118.45 \mathrm{~ms}$ & $89.00 \mathrm{~ms}$ & $248.78 \mathrm{~ms}$ & $59.40 \mathrm{~ms}$ \\
1021 & $72.00 \mathrm{~ms}$ & $143.10 \mathrm{~ms}$ & $74.50 \mathrm{~ms}$ & $77.00 \mathrm{~ms}$ & $105.73 \mathrm{~ms}$ & - & $61.44 \mathrm{~ms}$ & $213.09 \mathrm{~ms}$ & $111.22 \mathrm{~ms}$ \\
1047 & $57.82 \mathrm{~ms}$ & $162.00 \mathrm{~ms}$ & $45.57 \mathrm{~ms}$ & $58.42 \mathrm{~ms}$ & $80.00 \mathrm{~ms}$ & $23.89 \mathrm{~ms}$ & - & $219.56 \mathrm{~ms}$ & $41.30 \mathrm{~ms}$ \\
1051 & $153.20 \mathrm{~ms}$ & $100.70 \mathrm{~ms}$ & $71.30 \mathrm{~ms}$ & $36.40 \mathrm{~ms}$ & $177.00 \mathrm{~ms}$ & $100.00 \mathrm{~ms}$ & $104.20 \mathrm{~ms}$ & - & $106.00 \mathrm{~ms}$ \\
1080 & $73.91 \mathrm{~ms}$ & $118.33 \mathrm{~ms}$ & $64.11 \mathrm{~ms}$ & $163.13 \mathrm{~ms}$ & $117.50 \mathrm{~ms}$ & $119.27 \mathrm{~ms}$ & $59.89 \mathrm{~ms}$ & $140.60 \mathrm{~ms}$ & - \\
base & $92.55 \mathrm{~ms}$ & $114.30 \mathrm{~ms}$ & $89.80 \mathrm{~ms}$ & $88.64 \mathrm{~ms}$ & $127.33 \mathrm{~ms}$ & $88.40 \mathrm{~ms}$ & $83.55 \mathrm{~ms}$ & $100.18 \mathrm{~ms}$ & $73.44 \mathrm{~ms}$ \\
\hline
\end{tabular}

The results show a significant number of errors where either packets were lost or packets got duplicated somewhere in the network. This is possible because by using the UDPEcho application, the packets are not only sent point-to-point between two MAC layer instances as for the RSSI analysis but rather are allowed to use multi-hop communication thanks to the employed routing protocol. Besides the additional duplicates and errors, the delay is significantly higher in this setup which was again rather unexpected after the previous results. Furthermore, it shows a high variance between individual links. Using the multicast address might have caused additional collisions or backoff times at the MAC layer and thus explains the additional loss and delay variations as compared to the measurements performed via the PC.

While all nodes show a quite good connection to the base station, the connectivity between different node pairs is quite diverse and shows highly asymmetric behavior. Still, all nodes are able to connect to each other and thus form a star topology. However, due to the difficult propagation conditions for some nodes, this star is changed adaptively to a tree-like structure based on the routing protocol.

As in the previous measurements, the nodes of the exterior subsystem show again a worse performance when connecting to nodes positioned within the car and a slightly better performance when connecting to fellow nodes of that subsystem. The results shown in Table IV combined with the RSSI values presented in Figure IV-C indicate the difficulty to communicate through the body of the car using wireless transmissions.

Our results give some insights on the deployment options of wireless sensor networks for intra-vehicle monitoring and clearly indicate potential problems even though the measurements were performed under rather good conditions in a parking lot. Highly dynamic propagation characteristics make any communication from the outside through the body of the car challenging.

The number of sensors supported by one base station and thus by one wireless channel might be limited if a setup similar to our base station consisting of a gateway/PC combination is used to access the sensor network. Such a base station was not able to handle even relatively small amounts of packets in a proper way without introducing an unacceptable additional delay and thus acted as a bottleneck. Therefore, multiple base stations should be used in order to limit the traffic that one base station has to handle and at the same time to reduce the distance that packets have to travel over-the-air. However, simply introducing additional base stations might not solve the problem completely as the already observed duplicates show. If multiple base stations are used, a significant amount of traffic can be received by both, if the same channel is used. This would increase the probability of duplicated packets at the wired side and thus needs additional duplicate handling. One should keep in mind, that our results are obtained under idealized conditions. More realistic scenarios will most likely cause further performance reduction due to more complex propagation conditions and channel characteristics.

Besides that, the tested sensor nodes use the default IEEE 802.15.4 Carrier Sense Multiple Access / Collision Avoidance (CSMA/CA) medium access scheme resulting in large delay variations in case of high medium utilization caused by multiple nodes accessing the channel. This corresponds to the results in [15]. Other MAC-schemes might show a better and more predictable performance that would be required for control applications. Especially the Time Division Multiple Access (TDMA) extensions [16], [17] described in the recent standard document [18] are interesting for this and first implementations are also presented recently (e.g. in [19]).

For low-rate interior applications like passenger detection or air-conditioning monitoring and control, the given technology is sufficient because the sensor values change infrequently and do not have any real-time constraints.

\section{CONClusion And Future Work}

In this paper, we presented results from measurements performed with custom sensor nodes inside a vehicle in order to evaluate the performance of such a network within a vehicle under idealized conditions and identify potential issues. The results showed that the existing protocol stack is able to handle 
TABLE V

In-Network ERror Rate (Duplicates AND LOSS COMBined) FOR ALl Node PERMUtATIONS

\begin{tabular}{crrrrrrrrrr}
\hline destination & \multicolumn{10}{c}{ sender } \\
\cline { 2 - 11 } node ID & 1001 & 1002 & 1008 & 1009 & 1010 & 1021 & 1047 & 1051 & 1080 \\
\hline 1001 & - & 0.3 & 0.1 & 0.0 & 0.4 & 0.2 & 0.2 & 0.2 & 0.3 \\
1002 & 0.2 & - & 0.1 & 0.0 & 0.1 & 0.1 & 0.0 & 0.0 & 0.1 \\
1008 & 0.2 & 0.2 & - & 0.1 & 0.2 & 0.0 & 0.0 & 0.3 & 0.3 \\
1009 & 0.2 & 0.1 & 0.1 & - & 0.0 & 0.1 & 0.0 & 0.1 & 0.1 \\
1010 & 0.3 & 0.2 & 0.1 & 0.1 & - & 0.3 & 0.0 & 0.1 & 0.0 \\
1021 & 0.1 & 0.2 & 0.2 & 0.1 & 0.1 & - & 0.1 & 0.1 & 0.2 \\
1047 & 0.1 & 0.1 & 0.3 & 0.3 & 0.1 & 0.1 & - & 0.1 & 0.1 \\
1051 & 0.2 & 0.0 & 0.0 & 0.0 & 0.1 & 0.1 & 0.1 & - & 0.1 \\
1080 & 0.2 & 0.2 & 0.1 & 0.2 & 0.0 & 0.5 & 0.1 & 0.2 & - \\
base & 0.1 & 0.0 & 0.0 & 0.1 & 0.2 & 0.0 & 0.1 & 0.1 & 0.1 \\
\hline
\end{tabular}

low data rates quite well, if no strict timing constraints apply. Even if RSSI values indicate a rather poor channeel, the delivery ratio is high indicating that the employed protocols are able to handle the challenging propagation conditions.

But the picture is somewhat different for more dense networks, networks with high packet rates, and networks with frequent parallel transmissions. In these three cases, the used setup with a gateway node connected via a serial interface acted as bottleneck and causing unacceptable delays. Therefore, single bottlenecks should be avoided by deploying multiple gateways and potentially using separate channels for different subsystems for better traffic separation. However, such an approach does not scale well as the number of channels is limited. Therefore, we want to exploit the impact of especially customized MAC protocol versions in the future in addition to further measurements under more realistic scenarios as well as finer sampling and arbitrary data generation rates.

Besides that, we want to analyze interferences and security issues due to the limited channel availability. If multiple vehicles are equipped with sensor nodes, the chances are high that nodes deployed on neighboring vehicles are within communication range and thus could potentially receive messages intended for the other vehicle's network as shown by the measurements for exterior to interior communication.

\section{REFERENCES}

[1] H. Wymeersch, G. R. de Campos, P. Falcone, L. Svensson, and E. G. Ström, "Challenges for Cooperative ITS: Improving Road Safety Through the Integration of Wireless Communications, Control, and Positioning," in International Conference on Computing, Networking and Communications (ICNC). IEEE, Feb. 2015, pp. 573-578.

[2] J. Åkerberg, M. Gidlund, and M. Björkman, "Future Research Challenges in Wireless Sensor and Actuator Networks Targeting Industrial Automation," in 9th IEEE International Conference on Industrial Informatics (INDIN). IEEE, 2011, pp. 410-415.

[3] M. A. Rahman, "Design of Wireless Sensor Network for Intravehicular Communications," in Wired/Wireless Internet Communications. Springer, 2014, pp. 29-40.

[4] V. C. Gungor and G. P. Hancke, "Industrial Wireless Sensor Networks: Challenges, Design Principles, and Technical Approaches," IEEE Transactions on Industrial Electronics, vol. 56, no. 10, pp. 4258-4265, 2009.
[5] W. M. H. Heemels, A. R. Teel, N. van de Wouw, and D. Nesic "Networked Control Systems With Communication Constraints: Tradeoffs Between Transmission Intervals, Delays and Performance," IEEE Transactions on Automatic Control, vol. 55, no. 8, pp. 1781-1796, 2010.

[6] J. M. Winter, I. Muller, G. Soatti, S. Savazzi, M. Nicoli, L. B. Becker, J. C. Netto, and C. E. Pereira, "Wireless Coexistence and Spectrum Sensing in Industrial Internet of Things: An Experimental Study," International Journal of Distributed Sensor Networks, vol. 11, 2015.

[7] R. M. Pellegrini, S. Persia, D. Volponi, and G. Marcone, "RF propagation analysis for ZigBee Sensor Network using RSSI measurements," in 2nd International Conference on Wireless Communication, Vehicular Technology, Information Theory and Aerospace \& Electronic Systems Technology (Wireless VITAE). IEEE, 2011, pp. 1-5.

[8] A. Kadri, "Performance of IEEE 802.15. 4-based wireless sensors in harsh environments," in 8th International Wireless Communications and Mobile Computing Conference (IWCMC). IEEE, 2012, pp. 526-530.

[9] J.-R. Lin, T. Talty, and O. K. Tonguz, "An empirical performance study of Intra-vehicular Wireless Sensor Networks under WiFi and Bluetooth interference," in Global Communications Conference (GLOBECOM). IEEE, 2013, pp. 581-586.

[10] M. Hashemi, W. Si, M. Laifenfeld, D. Starobinski, and A. Trachtenberg, "Intra-car Wireless Sensors Data Collection: A Multi-hop Approach," in 77th Vehicular Technology Conference (VTC Spring). IEEE, 2013, pp. $1-5$.

[11] S. Engelhardt, E. Chervakova, A. Schreiber, T. Rossbach, W. Kattanek, and M. Götze, "BASe-Kit-Ein mobiles Messsystem für die Gebäudeautomation," 15. ITG/GMA-Fachtagung Sensoren und Messsysteme, pp. 431-435, 2010, in German.

[12] TinyOS Documentation Wiki. [Online]. Available: http://tinyos.stanford.edu/tinyos-wiki/index.php

[13] M. Götze, W. Kattanek, and R. Peukert, "An Extensible Platform for Smart Home Services," in 9th International Multi-Conference on Systems, Signals and Devices (SSD). IEEE, 2012.

[14] A. Tavakoli, J. Hui, and D. Culler, "HYDRO: A Hybrid Routing Protocol for Lossy and Low Power Networks," March 2009. [Online]. Available: http://tools.ietf.org/pdf/draft-tavakoli-hydro-01.txt

[15] M. Doudou, D. Djenouri, and N. Badache, "Survey on Latency Issues of Asynchronous MAC Protocols in Delay-Sensitive Wireless Sensor Networks," Communications Surveys \& Tutorials, vol. 15, no. 2, pp. 528-550, 2013.

[16] W.-C. Jeong and J. Lee, "Performance Evaluation of IEEE 802.15.4e DSME MAC Protocol for Wireless Sensor Networks ," in 1st Workshop on Enabling Technologies for Smartphone and Internet of Things (ETSIoT). IEEE, 2012, pp. 7-12.

[17] D. Dujovne, T. Watteyne, X. Vilajosana, and P. Thubert, "6TiSCH: Deterministic IP-Enabled Industrial Internet (of Things)," IEEE Communications Magazine, vol. 52, no. 12, pp. 36-41, 2014.

[18] "IEEE Standard for Low-Rate Wireless Networks," IEEE Std 802.15.42015, April 2016.

[19] M. Köstler, F. Kauer, T. Lübkert, and V. Turau, "Towards an Open Source Implementation of the IEEE 802.15.4 DSME Link Layer," in 15. GI/ITG KuVS Fachgespräch Sensornetze (FGSN), Augsburg, Germany, Sept. 2016, pp. 31-34. 


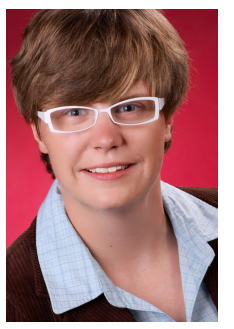

Silvia Krug (Research Fellow - Communication Networks Group, Technische Universität Ilmenau) received her M.Sc. degree from Technische Universität Ilmenau in January 2013. In April 2013, she joined the Communication Networks Group at Technische Universität Ilmenau as doctoral researcher. Her main research focus are delay tolerant networking in disaster scenarios and wireless sensor networks as well as embedded systems.



Hannes Toepfer (Professor - Advanced Electromagnetics Group, Technische Universität Ilmenau) studied Electrical Engineering at the Institute of Technology Ilmenau. From 1991-2002 he worked as an scientific assistant at the Ilmenau University of Technology. In 1996, he received the Dr.-Ing. (equiv. $\mathrm{PhD})$ and in 2003 his postdoctoral qualification (Habilitation). In 2002, he joined the Institute for Microelectronics and Mechatronics Systems where he has been directing the System Design department. In 2009, he became a Full Professor at Technische Universität Ilmenau and heading the Advanced Electromagnetics Group with a research focus on electromagnetic sensors.

$\mathrm{He}$ is a senior member of the IEEE.



Tino Hutschenreuther (Manager - Dept. System Design IMMS) studied computer science at the Dresden University of Technology. From 1994-1999 he worked as a research assistant at the Dresden University of Technology in computer networks. From 2000 to 2009 he worked as a consultant and technical project manager for IT projects in the logistics field. In 2000, he received his doctorate with a dissertation on routing protocols in IP networks. In 2009, he joined the Institute for Microelectronics and Mechatronics Systems, a non-profit organization in Ilmenau, where he directs the System Design department with the research focus on embedded systems and sensor networks.

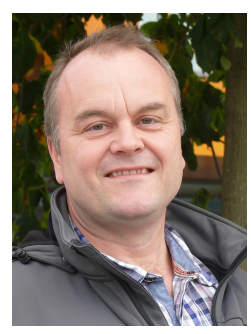

Jochen Seitz (Professor - Communication Networks Group, Technische Universität Ilmenau) studied computer science at Universität Karlsruhe (TH) and received his $\mathrm{PhD}$ from the same University in 1993 in the area of network management. After a postdoc year at Lancaster University, United Kingdom, and his postdoctoral qualification (Habilitation), he became a professor of Communication Networks at Technische Universität Ilmenau in 2001. 